\title{
PRODUÇÃO INTEGRADA E SEGURANÇA ALIMENTAR NAS VILAS PRODUTIVAS RURAIS DO PROJETO DE INTEGRAÇÃO DO RIO SÃO FRANCISCO, VILA PRODUTIVA QUIXERAMOBIM NO MUNICÍPIO DE SÃO JOSÉ DE PIRANHAS - PARAÍBA
}

\author{
INTEGRATED PRODUCTION AND FOOD SAFETY IN THE \\ RURAL PRODUCTIVE VILLAGES OF THE SÃO FRANCISCO \\ RIVER INTEGRATION PROJECT, QUIXERAMOBIM \\ PRODUCTIVE VILLAGE IN THE MUNICIPALITY OF SÃO JOSE \\ DE PIRANHAS - PARAÍBA
}

\author{
Andréa Araújo de Aquino \\ Universidade Federal do Vale do São Francisco, Brasil. \\ aquinoandrea257@gmail.com \\ Braz José do Nascimento Júnior \\ Universidade Federal do Vale do São Francisco, Brasil. \\ braz.jose@univasf.edu.br \\ Luciana Sousa de Oliveira \\ Instituto Federal do Serão Pernambucano \\ luciana.ifsertaope@gmail.com
}

\section{Resumo}

A reinserção socioprodutiva das famílias reassentadas pelo Projeto de Transposição do Rio São Francisco - PISF é um dos grandes desafios enfrentados nas Vilas Produtivas Rurais do projeto. O objetivo desse relato é apresentar a experiência de uma família na vila produtiva Quixeramobim no município de São Jose de Piranhas - PB, que desenvolve em seu quintal produtivo praticas agroecológicas de produção, na perspectiva da segurança alimentar e geração de renda. No contexto do semiárido nordestino, o Programa Básico Ambiental (PBA 08), desenvolvido pela Universidade Federal do Vale do São Francisco - UNIVASF em parceria com o Ministério do Desenvolvimento Regional - MDA, propôs processos sociais locais que contribuíssem com a segurança alimentar, geração de ocupação e renda e autonomia das famílias reassentadas do PISF. Através de Metodologias Participativas (Linha do Tempo, Caminhada transversal e FOFA) os agricultores familiares indicaram os cursos de Quintal Produtivo e Horta Agroecológica como meios de desenvolver sistemas produtivos adaptados à convivência com o Semiárido em consonância com a preservação do meio ambiente. 
Produção integrada e segurança alimentar nas vilas produtivas rurais do projeto de integração do Rio São Francisco, vila produtiva Quixeramobim no município de São Jose de Piranhas - Paraíba
Andréa Araújo de Aquino

Braz José do Nascimento Júnior

Luciana Sousa de Oliveira

Palavras-Chave: Segurança Alimentar. Horta Agroecológica. Reinserção Produtiva. Geração de Renda.

\begin{abstract}
The socio-productive reinsertion of families resettled by the São Francisco River Transposition Project - PISF, is one of the great challenges faced in the Rural Productive Villages of the project. The objective of this article is to present the experience of a family in the Quixeramobim productive village in the municipality of São José de Piranhas - PB, which develops agroecological practices of production in its productive backyard, from the perspective of food security and income generation. In the context of the semi-arid region of the Northeast, the Basic Environmental Program (PBA 08), developed by the Federal University of Vale do São Francisco - UNIVASF in partnership with the Ministry of Regional Development - MDA, proposed local social processes that contribute to food security, generation of occupation and income and autonomy of resettled families from the PISF. Through Participatory Methodologies (Timeline, Cross-walk and SWOT) family farmers indicated the courses of Productive Backyard and Agroecological Garden as means of developing production systems adapted to coexistence with the Semiarid region, in line with the preservation of the environment.
\end{abstract}

Keywords: Food Secury. Agroecological. Productive Reinsertion. Income Generation.

\title{
Introdução
}

Para construção da obra da transposição do Rio São Francisco (PISF) cerca 3.500 pessoas, totalizando 845 famílias, tiveram que ser retiradas do lugar onde viviam, deixando para trás seus costumes, organizações sociais e meios de produção destinados à alimentação e a geração de renda (RIMA, 2004; BORGES, 2013).

Diante disto foram criados 38 Programas Básicos Ambientais (PBAs), entre os quais, o PBA 8, que estabelece ações de reassentamento de 848 famílias em 18 Vilas Produtivas Rurais (VPRs), denominadas como: Captação, Baixio dos Grandes, Negreiros, Uri, Queimada Grande, Malicia, Pilões e Salão, no estado de Pernambuco; Ipê, Retiro, Descanso e Vassouras, no estado do Ceará; e Cacaré, Quixeramobim, Bartolomeu, Lafayette, Irapuá I e II, na Paraíba. Todas construídas ao longo da faixa de 2,5 km a partir de cada uma das margens dos canais, como forma de potencializar as atividades produtivas a serem desenvolvidas pela população reassentada (BORGES, 2013). 
Produção integrada e segurança alimentar nas vilas produtivas rurais do projeto de integração do Rio São Francisco, vila produtiva Quixeramobim no município de São Jose de Piranhas - Paraíba
Andréa Araújo de Aquino Braz José do Nascimento Júnior Luciana Sousa de Oliveira

Cada família vive com uma renda provisória de um salário mínimo por mês até a primeira produção, garantida na época pelo Ministério da Integração - MI e assumida atualmente pelo Ministério do Desenvolvimento Regional - MDR. Além disso, todas tiveram direito a uma casa de $99 \mathrm{~m}^{2}$, inserida em um lote residencial de 0,5 hectares e mais cinco hectares destinados às atividades agropecuárias, sendo quatro de sequeiro e um que será irrigado.

A ação do PBA 8 tem como objetivo principal propiciar às famílias afetadas condições que permitam sua reprodução social e econômica, em situação minimamente similar à que viviam antes de serem reassentadas nas VPRs (RIMA, 2004, p. 99).

Neste sentido, foi realizada uma parceria entre o MDR e a Universidade Federal do Vale do São Francisco - UNIVASF para a realização de cursos de capacitação destinados principalmente a promoção do Desenvolvimento Rural Sustentável tendo como princípios a promoção de processos sociais locais que contribuam com a segurança alimentar, geração de ocupação e renda visando promover a autonomia financeira e alimentar das famílias, além do apoio aos processos de organizações associativas locais e a constituição de sistemas produtivos adaptados à convivência com o Semiárido em consonância com a preservação do meio ambiente.

$\mathrm{O}$ contexto climático e ambiental no semiárido nordestino impõe às comunidades rurais a busca por alternativas produtivas para sua sobrevivência e desenvolvimento.

Silva (2006) afirma que a convivência com o semiárido não se trata simplesmente de novas técnicas, de novas atividades e práticas produtivas, de ações socioculturais etc. A convivência com o semiárido é uma proposta cultural, que visa contextualiza saberes e práticas (tecnológicas, econômicas e políticas) apropriado à semi-aridez, reconhecendo a sua heterogeneidade de suas manifestações, considerando também as compreensões imaginarias da população local sobre esse espaço, suas problemáticas e alternativas de solução.

Dessa forma, foram desencadeadas em todas as vilas produtivas do PISF atividades de caráter coletivo com oficinas de planejamento social e cursos de capacitação em sistemas de produção integrados a partir do desenvolvimento de metodologias participativas. O presente trabalho visa sistematizar, apresentar e discutir os resultados a partir da experiência do cultivo de horta agroecológica no contexto de 
Produção integrada e segurança alimentar nas vilas produtivas rurais do projeto de integração do Rio São Francisco, vila produtiva Quixeramobim no município de São Jose de Piranhas - Paraíba
Andréa Araújo de Aquino

Braz José do Nascimento Júnior

Luciana Sousa de Oliveira

um quintal produtivo desenvolvido na VPR Quixeramobim, localizada no Município de

São José de Piranhas, Estado da Paraíba.

\section{Metodologia}

Por meio de uma intervenção participativa, estruturada desde análise do Plano de Desenvolvimento Sustentável - PDS, passando pelo curso de organização e planejamento até a realização dos cursos produtivos, a metodologia fundamentou-se no Relato de Experiência (RE).

Para Daltro e Faria (2019), o RE é resultante de um processo; melhor dizendo, pode-se considerá-lo em um entrecruzamento de processos, dos coletivizados aos mais singulares. No tocante aos processos singulares...

[...] é importante salientar que o RE poderá ser conhecido como de algo que o impactou. Pressupõe-se no RE um trabalho de concatenação e memória, a elaboração de um acontecido que como vê seu relator, invocando suas competências reflexivas e associativas, bem como suas crenças e posições de sujeito no mundo. Então, o trabalho narrativo da singularidade no RE é sempre e invariavelmente um trabalho de linguagem. Já os aspectos coletivizados, colocam ao pesquisador o desafio de articular teoricamente conhecimentos que marcam seu pertencimento coletivo, ao mesmo tempo em que ativam suas competências de tradução, percepção e interpretação. (DALTRO; FARIA, 2019, p. 226).

O curso de quintal produtivo na VPR Quixeramobim foi realizado no período de 21 a 25 de outubro de 2019 e teve carga horária de 40 horas, e contou com a participação de 19 reassentados, a ideia era que a equipe auxiliasse as famílias a definirem os temas considerados prioritários, de acordo com suas atividades produtivas. Para isso, foram utilizadas as seguintes metodologias participativas: linha do tempo (resgate histórico), caminhada transversal na comunidade, matriz FOFA (Fortaleza, Oportunidade, Fraqueza e Ameaça) e matriz de prioridades.

Para Ribeiro e Marinho (2015) é possível compreender as Metodologias Participativas como instrumentos fundamentais para promoção da participação popular, com vistas na construção de novos conhecimentos necessários para sustentabilidade socioambiental, à promoção da Agroecologia e da agricultura familiar junto à diversidade de grupos e atores sociais do/no campo. 
Produção integrada e segurança alimentar nas vilas produtivas rurais do projeto de integração do Rio São Francisco, vila produtiva Quixeramobim no município de São Jose de Piranhas - Paraíba
Andréa Araújo de Aquino Braz José do Nascimento Júnior Luciana Sousa de Oliveira

Os resultados do Curso de Organização e Planejamento Socioambiental na VPR Quixeramobim direcionou inicialmente a equipe do PBA 8 para a realização de dois cursos: Quintal Produtivo e Produção Agroecológicas Comunitária de Hortaliças. Nesse sentido, entende-se que os cursos se complementavam em conteúdo e metodologia.

Costa e Strate (2018) indicam que os quintais produtivos são uma das formas mais antigas de manejo da terra e consistem em uma combinação de espécies florestais, agrícolas, medicinais e ornamentais, algumas vezes associado à pequena criação de animais domésticos, ao redor da residência. Essa prática tem contribuído de forma efetiva para segurança alimentar.

Para Barbosa (2013), o sistema socioprodutivo agroecológico pode constitui-se numa alternativa para construção de um processo de desenvolvimento para o ambiente rural que se estrutura por meio de particularidades social, econômica, cultural e ecológica existentes nesse ambiente.

\section{VPR Quixeramobim}

A VPR Quixeramobim é uma das cinco vilas produtivas rurais do PISF no estado da Paraíba, localizada no município de São José de Piranhas região geográfica imediata de Cajazeiras no semiárido paraibano, foi criada em 07 de março de 2016, inicialmente com 47 famílias reassentadas. O acesso a VPR é realizado pela rodovia estadual PB-366, estando 26 km de distância da sede municipal.

O setor produtivo é constituído por lotes de sequeiro com área média individual de aproximadamente 6,0 ha e lotes irrigáveis de aproximadamente 1,00 ha. A área destinada à instalação de infraestrutura coletiva de apoio à produção de uso coletivo é de 225,29664 hectares (MDR, 2019). 
Produção integrada e segurança alimentar nas vilas produtivas rurais do projeto de integração do Rio São Francisco, vila produtiva Quixeramobim no município de São Jose de Piranhas - Paraíba
Andréa Araújo de Aquino Braz José do Nascimento Júnior

Luciana Sousa de Oliveira

Figura 1. Mapa de Arranjo Geral do Setor Residencial da VPR Quixeramobim

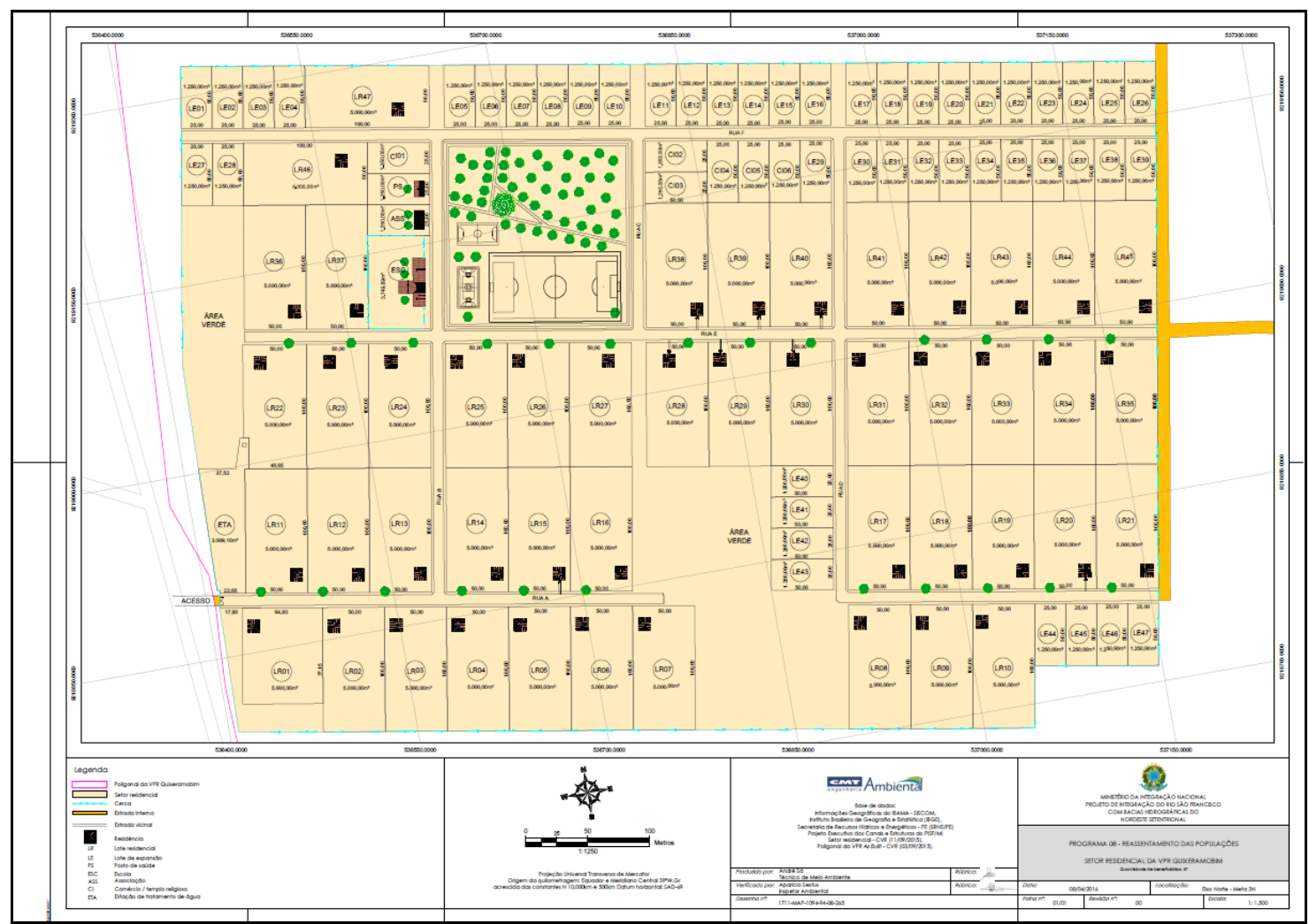

\section{Resultado e Discussão}

\section{Curso de Quintal Produtivo}

Esse curso foi realizado nos quintais residenciais das famílias, com carga horária de 40 horas, no período de 21 a 25 de outubro de 2019, com 40 participantes onde foram abordados os seguintes temas: convivência com o semiárido, formas de captação de água para produção, reutilização de águas cinzas, produção de compostos orgânicos, preparação caldas biofertilizantes e defensivos naturais, confecção de canteiros, adubação e irrigação, estrutura para instalação de aviário e alimentação alternativa para criação de galinhas. 
Produção integrada e segurança alimentar nas vilas produtivas rurais do projeto de integração do Rio São Francisco, vila produtiva Quixeramobim no município de São Jose de Piranhas - Paraíba
Andréa Araújo de Aquino Braz José do Nascimento Júnior

Luciana Sousa de Oliveira

Figura 3: Produção de compostagem, biofertilizantes e defensivos naturais.

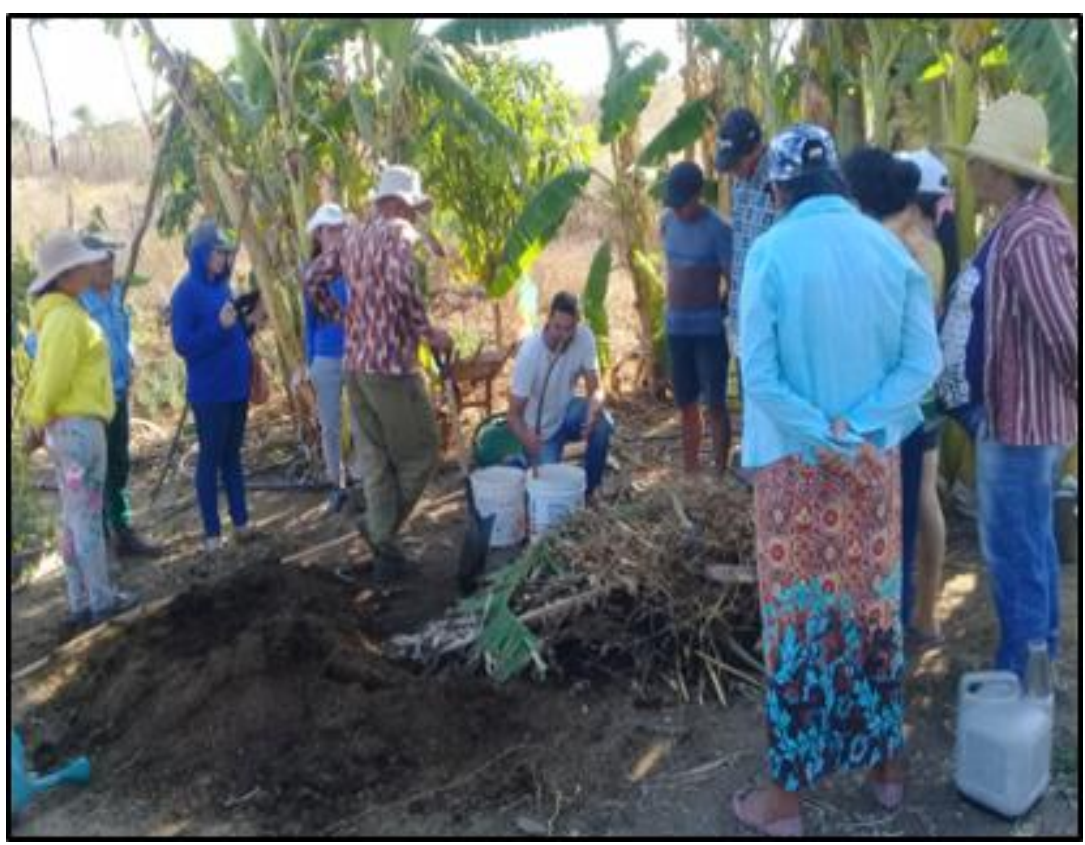

Fonte: Autor, 2021.

\section{Curso de Produção Agrológica de Hortaliças Comunitária}

Realizado no período de 06 a 10 de janeiro de 2020. Esse curso teve carga horária de 40 horas e foi realizado em área coletiva. Os temas abordados foram: diferença entre agricultura orgânica e convencional; critérios que devem ser observados na instalação de uma horta comunitária; produção de compostos orgânicos; preparação caldas biofertilizantes; confecção de canteiros e adubação; irrigação; estruturas utilizadas na produção de mudas; preparação de defensivos naturais e confecção de armadilhas.

Após a realização dos cursos de quintal e horta, a família da agricultora optou por iniciar a produção de horta agroecológica em seu quintal residencial. Onde cultiva atualmente plantas frutíferas (banana, goiaba, maracujá) hortaliças (jerimum, pimentão, couve, coentro, cebolinha, alface, pimenta, tomate cereja), gramíneas (cana, milho), tubérculos (macaxeira) e aves (galinhas caipiras). 
Produção integrada e segurança alimentar nas vilas produtivas rurais do projeto de integração do Rio São Francisco, vila produtiva Quixeramobim no município de São Jose de Piranhas - Paraíba
Andréa Araújo de Aquino Braz José do Nascimento Júnior

Luciana Sousa de Oliveira

Figura 06 - Preparo da área para implantação da Horta Agroecológica.

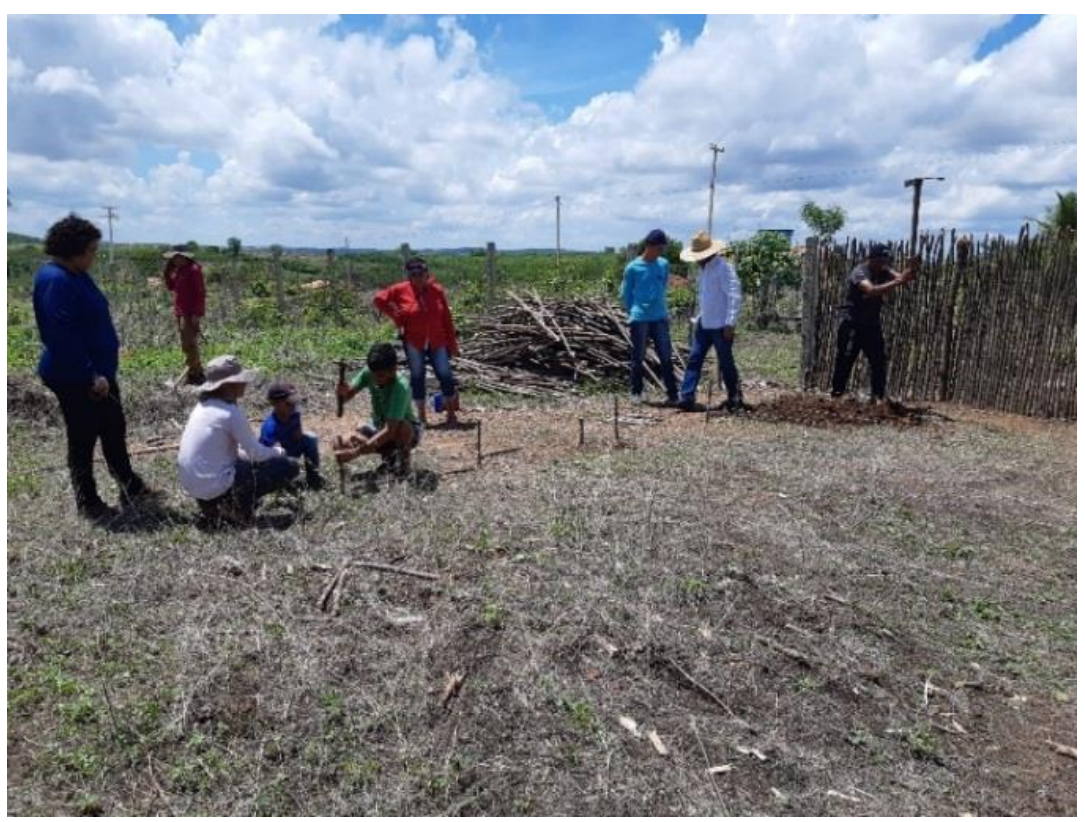

Fonte: Autor, 2021.

Figura 07 - Preparo dos canteiros e montagem do sistema de irrigação

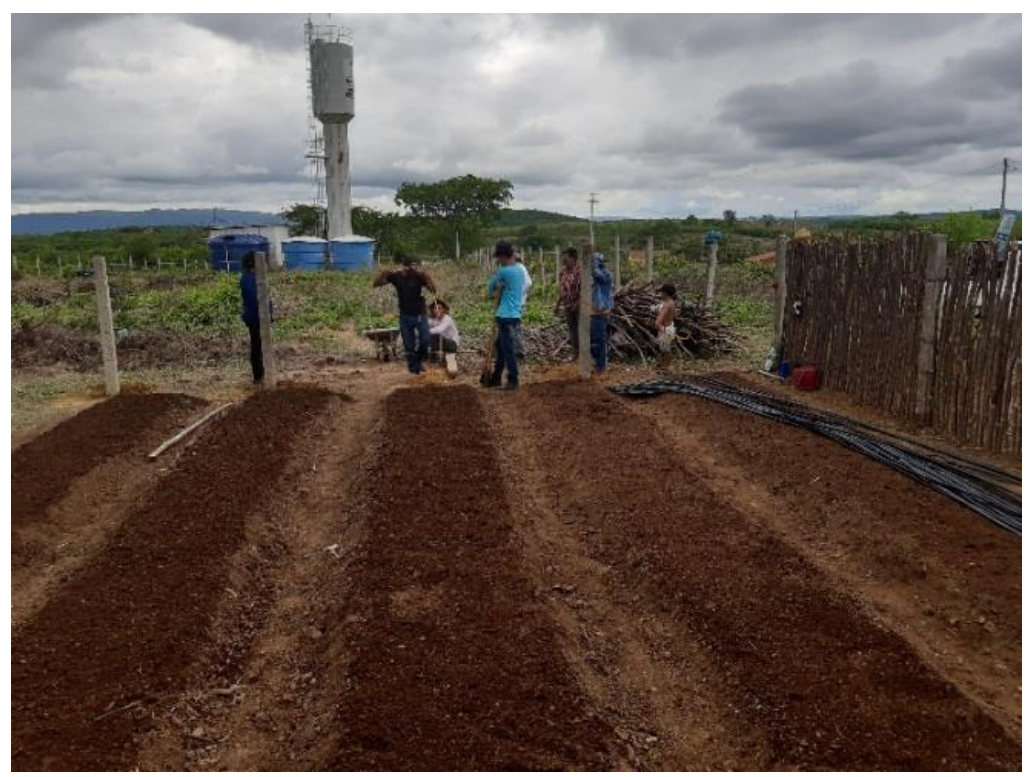

Fonte: Autor, 2021

Para proteção da horta a família utiliza da bucha-pepino (Luffa acutângula) e maracujá (Passiflora edulis) em volta da cerca do quintal. Além disso, é utilizada a tela sombrite para proteção contra o ataque de pássaros e a alta incidência da radiação solar. 
Produção integrada e segurança alimentar nas vilas produtivas rurais do projeto de integração do Rio São Francisco, vila produtiva Quixeramobim no município de São Jose de Piranhas - Paraíba
Andréa Araújo de Aquino Braz José do Nascimento Júnior Luciana Sousa de Oliveira

No cultivo do coentro, cebolinha e alface, condicionados em seis canteiros de 9 $\mathrm{m}$ de comprimento e 1,2m de largura, a família utiliza na adubação o esterco bovino curtido e um biofertilizante composto por pó de café e casca do ovo, que é aplicado no pé das plantas a cada 15 dias.

A agricultora conta que muitas das práticas que utiliza em seu quintal foram aprendidas durante os cursos, no entanto, com a pandemia não houve uma assessoria técnica adequada, além dos contatos por telefone com a equipe da UNIVASF, a agricultura também se utiliza das redes sociais como o Youtube para tirar dúvidas em relação aos manejos.

Outra dificuldade apontada pela agricultora é a escassez de água, uma vez que a única fonte de água disponível é um poço artesiano com uma vazão de 200 litros de água por hora e o sistema de abastecimento de água da comunidade, pelo qual paga uma taxa de $\mathrm{R} \$ 280,00$ mensais para utilização de 70 mil litros de água/mês.

Por mês a família produz em média 150 molhos de coentro/mês, 120 pés de alface/mês, e 100 molhos de cebolinha/mês.

Com essa produção a agricultora contou que consegue obter uma renda mensal de aproximadamente meio salário mínimo por mês.

Figura 8 - Plantio de Coentro Orgânico

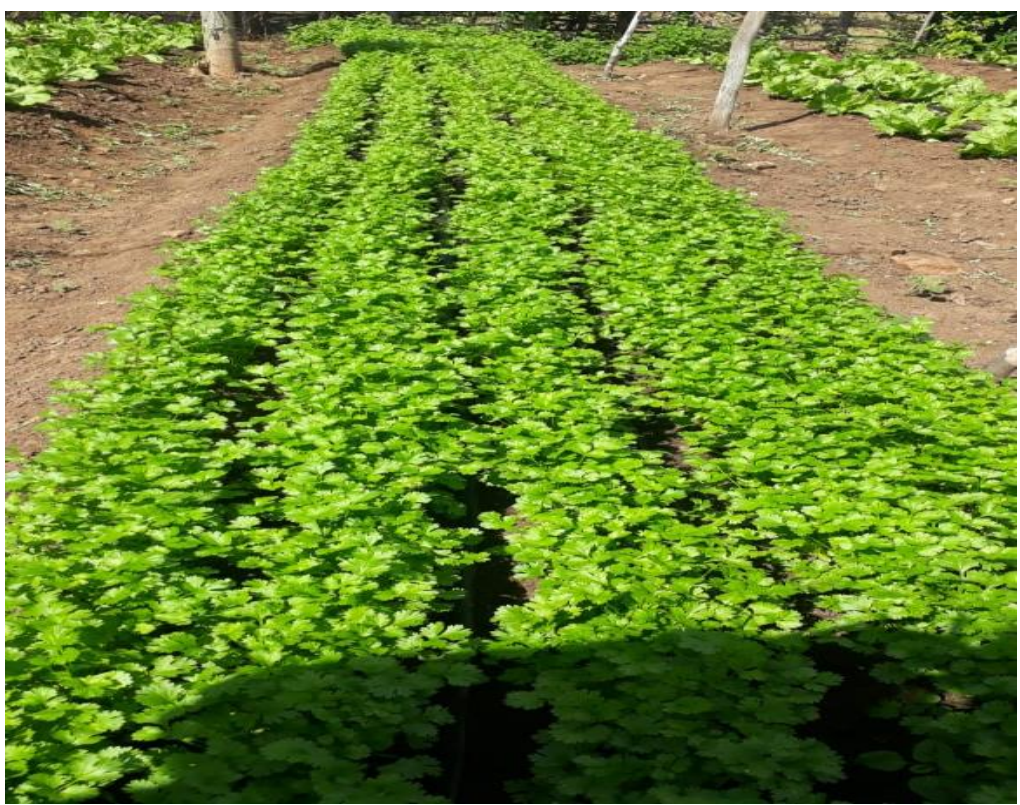

Fonte: Autor, 2021. 
Produção integrada e segurança alimentar nas vilas produtivas rurais do projeto de integração do Rio São Francisco, vila produtiva Quixeramobim no município de São Jose de Piranhas - Paraíba
Andréa Araújo de Aquino Braz José do Nascimento Júnior

Luciana Sousa de Oliveira

Figura 10 - Plantio de Cebolinha Orgânica

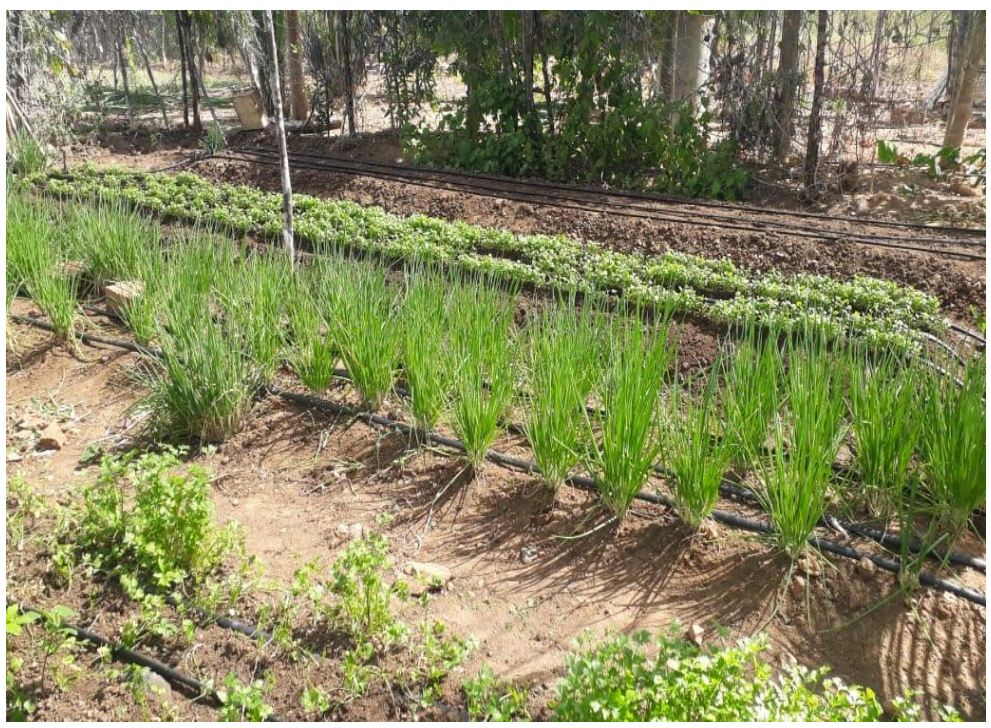

Fonte: Autor, 2021.

Em fevereiro de 2021 a família foi conseguiu entrar em um edital para a comercialização dos produtos através do Programa Nacional de Aquisição de Alimentos (PAA) e aguarda ansiosamente para dar início as vendas através deste programa, atualmente para comercializar seus produtos a agricultora conta que tira foto da produção, coloca no status do aplicativo WhatsApp para grupos e as pessoas fazem os pedidos.

Em vídeo enviado a equipe da UNIVASF, agricultora ressalta a importância dos processos propostos e as parcerias estabelecidas com outros atores sociais a partir das metodologias participativas que trouxeram uma visão mais ampla para a comunidade e os participantes dos cursos e diz que seria muito importante que tivesse outra capacitação voltada para a comercialização dos produtos.

\section{Considerações Finais}

A experiência da agricultora ressalta a importância da reinserção produtiva para famílias reassentadas do PISF, pois, embora, haja pouca água e falte assessoria técnica para as famílias, uma vez que a única assistência são as capacitações realizadas no PBA 8, a agricultura juntamente com sua família está mostrando que é possível produzir mesmo diante das dificuldades. 
Produção integrada e segurança alimentar nas vilas produtivas rurais do projeto de integração do Rio São Francisco, vila produtiva Quixeramobim no município de São Jose de Piranhas - Paraíba
Andréa Araújo de Aquino Braz José do Nascimento Júnior Luciana Sousa de Oliveira

As discussões propostas foram de caráter coletivo e proporcionaram uma construção pratica e participativa de incentivo ao uso de práticas agroecológicas junto às famílias das VPRs do PISF para que as mesmas pudessem desenvolver suas atividades de forma autônoma e na perspectiva do desenvolvimento sustentável e convivência com o semiárido

Durante todo o processo de organização e estruturação da horta comunitária os agricultores discutiram a importância de atividades coletivas na comunidade, o fortalecimento da associação enquanto instrumento facilitador para organização do grupo e sua representação para estruturação futura de uma cooperativa de comercialização da produção.

\section{REFERÊNCIAS}

BORGES, Fabiane Macedo. Associativismo no projeto São Francisco: estudo da Vila Produtiva Rural Negreiros. Sociedade em Debate, Pelotas, v. 19, n. 2, p. 209 - 235. 2013. Disponível em: https://revistas.ucpel.edu.br/rsd/article/view/959/744. Acesso em: 21 de dez. de 2021.

BARBOSA, Luciano Celso Brandão Guerreiro; BRANDENBURG, Alfio; LAGES, André Maia Gomes. A Pluriatividade na Agroecologia como uma Alternativa de Desenvolvimento para o Ambiente Rural. Revista Brasileira de Tecnologia Agropecuária, v. 1, n. 1, p. 86-96, 2017. Disponível em: http://revistas.fw.uri.br/index.php/rbdta/article/view/2426/2267. Acesso em: 21 de dez. de 2021.

DALTRO, M. R.; FARIA, A. A. Relato de experiência: Uma narrativa científica na pósmodernidade. Estudos \& Pesquisas em Psicologia, v. 19, n. 1, p. 223-237. 2019. Disponível em: https://www.epublicacoes.uerj.br/index.php/revispsi/article/view/43015/29726. Acesso em: 21 de dez. de 2021.

MARINHO, Cristiane Marinho; FREITAS, Helder Freitas. Utilização de Metodologias Participativas nos Processos de Assistência Técnica e Extensão Rural (ATER):

Fundamentos teórico-práticos. Extramuros, Petrolina-PE, v.3, n.3; p. 10-28, 2015. Disponível em: https://www.periodicos.univasf.edu.br/index.php/extramuros/article/view/764/526. Acesso em: 21 de dez. de 2021.

STRATE, M. F.; DA COSTA, S. M. Quintais Produtivos: contribuição à segurança alimentar e ao desenvolvimento sustentável das mulheres rurais no RS-Brasil. Brazilian Journal of development, Curitiba - PR, v.4, n. 7, p. 1-13, 2018. Disponível em: 
Produção integrada e segurança alimentar nas vilas produtivas rurais do projeto de integração do Rio São Francisco, vila produtiva Quixeramobim no município de São Jose de Piranhas - Paraíba
Andréa Araújo de Aquino Braz José do Nascimento Júnior Luciana Sousa de Oliveira

https://www.brazilianjournals.com/index.php/BRJD/article/view/387/331. Acesso em: 21 de dez. de 2021.

MINISTÉRIO DO DESENVOLVIMENTO REGIONAL - MDR. 38 Programas Ambientais - Programa de Reassentamento de Populações - PBA 08. Disponível em: https://antigo.mdr.gov.br/a-mudanca-em-sua-vida/meio-ambiente-preservado/38programas-ambientais. Acesso em: $21 \mathrm{dez}$. de 2021.

Ministério da Integração Nacional - MI. Plano de Trabalho - PBA 7 e 8 do PISF/UNIVASF. 2017. Disponível em: http://integracao.gov.br/documents/10157/4186139/Plano+de+Trabalho+-+TED+062017.pdf/b3067d31-3c35-4642-8881-8be6ebe51f0c. Acesso em: 21 de dez. de 2021.

RIMA - Relatório de Impacto Ambienta. Projeto de Integração do Rio São Francisco com Bacias Hidrográficas do Nordeste Setentrional. Ministério da Integração Nacional, 2004. 129 p. Disponível em:

https://antigo.mdr.gov.br/images/stories/ProjetoRioSaoFrancisco/ArquivosPDF/docume ntostecnicos/RIMAJULHO2004.pdf. Acesso em: 21 de dez. de 2021.

SILVA, Roberto Marinho Alves da. Entre o Combate à Seca e Convivência com o Semiárido: transições paradigmas e sustentabilidade do desenvolvimento. 2006. 226 f. Tese (Doutorado em Desenvolvimento Sustentável) - Universidade de Brasília, Centro de Desenvolvimento Sustentável, Brasília, 2011. Disponível em: https://repositorio.unb.br/bitstream/10482/2309/1/2006_Roberto\%20Marinho\%20Alves $\% 20 \mathrm{da} \% 20$ Silva.pdf. Acesso em: 21 de dez. de 2021.

Recebido em 21/06/2021.

Aceito para publicação em 18/12/2021. 